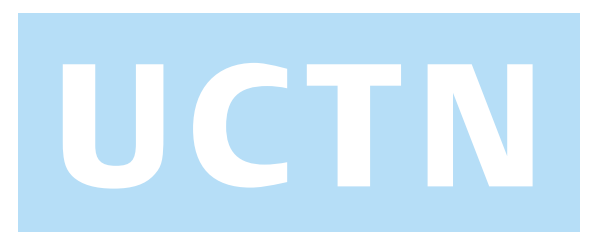

In September 1999, a 50-year-old man developed watery diarrhea with occasional hematochezia and abdominal cramps. The average stool frequency was about ten times in $24 \mathrm{~h}$. When first seen as an outpatient 6 weeks later, he had lost $10 \mathrm{~kg}$ in weight and had tachypnea (24/ min), hypotension $(80 \mathrm{mmHg}$, systolic) and tachycardia (100/min). Elevated erythrocyte sedimentation rate, marked anemia (hemoglobin $8 \mathrm{~g} / \mathrm{dl}$ ) and hypoproteinemia ( $3 \mathrm{~g} / \mathrm{dl}$ ) with edema of the lower limbs were present. The microbiological and parasitological stool examination were normal. Abdominal ultrasonography showed marked thickening of the hypoechoic colonic wall (12 mm), with loss of layering in the left hemicolon, whereas the remainder of the colon and the small bowel appeared normal. On colonoscopy (which terminated at the left flexure due to the patient's condition), severe ulcerative colitis was seen. However, the macroscopic picture was atypical for inflammatory bowel disease or any type of infectious enterocolitis. In the rectosigmoid, multiple small nodular lesions with occasional mucosal pinpoint-like hemorrhage were seen. In the descending colon, there

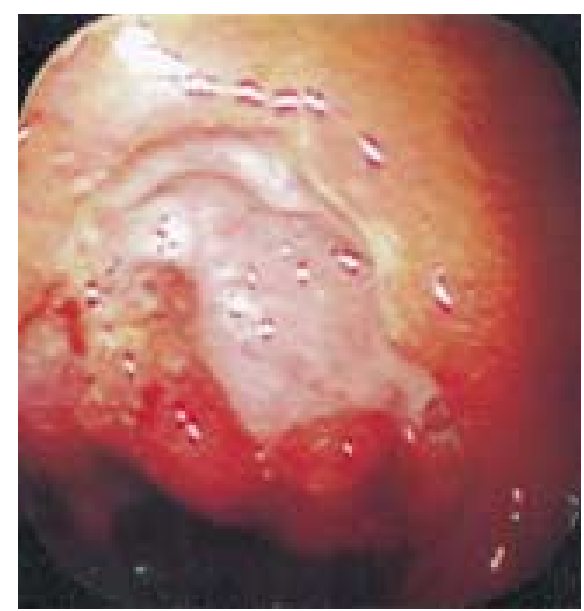

Figure 1 Endoscopic appearance of a representative lesion in the descending colon. A deep ulceration, partially covered by fibrin and surrounded in a circular fashion by protruding tissue, was observed

\title{
A Rare but Fatal Cause of Ulcerative Colitis
}

were deep ulcerations partially covered by fibrin and surrounded in a circular fashion by protruding tissue (Figure $\mathbf{1}$ ). The histopathological findings were compatible with unclassified colitis. The ulcerations extended beyond the mucosa, and the pathologist was unable to establish a definitive diagnosis.

The patient refused hospital admission, and was treated with prednisolone (50 mg/day) and sulfasalazine. After initial improvement, his condition deteriorated within 4 days, at which point hospitalization was required. Shortly afterwards, the patient stated that he had applied rectal instillations of hot water $\left(60^{\circ} \mathrm{C}\right)$, mixed with alkaline components such as soap or caustic soda, as part of masochistic sexual habits. Shortly after admission, the patient developed massive lower gastrointestinal bleeding caused by an anal ulcer, requiring multiple transfusions and several surgical interventions. During the second intervention, a perianal abscess was incised. Subsequently, the patient experienced a complicated septic course of the condition, with prolonged mechanical ventilation, neuropathy, and multiple-organ failure, and he finally died 6 months later.

In summary, this report describes a rare but fatal cause of ulcerative colitis, initially suggesting unclassified chronic inflammatory bowel disease. To the best of our knowledge, only one comparable case [1] has previously been reported. Self-induced colitis should be included in the differential diagnosis of unclassified colitis, especially when it is left-sided. However, the diagnosis can only be established by taking a thorough medical history, sometimes repeatedly.

\section{Folwaczny, K. Loeschke, M. Schmidt-Achert}

Dept. of Medicine, City Hospital, University of Munich, Munich, Germany

\section{Reference}

${ }^{1}$ Schapira M, Gerard D, Deltenre P et al. An unusual cause for left-sided colitis: hot water enema. Acta Gastroenterol Belg 1996; 59: 220-221

\section{Corresponding Author}

\section{Folwaczny, M.D.}

Medizinische Klinik Innenstadt Ludwig-Maximilians-Universität München

Ziemssenstrasse 1

80336 München

Germany

Fax: $\quad$ + 49-89-5160-4187

E-mail: Christian.Folwaczny@medinn. med.uni-muenchen.de 